Check for updates

Cite this: Mater. Adv., 2020, 1,1232

\title{
Electrodeposited ZnO nanoparticles on vertically aligned carbon nanotubes (VACNTs) as promising charge extracting electrodes for halide perovskite devices $\dagger$
}

\author{
Raphaëlle Belchi, ${ }^{a b}$ Baptiste Pibaleau, ${ }^{a}$ Mathieu Pinault, ${ }^{a}$ Bernard Ratier, ${ }^{b}$ \\ Nathalie Herlin-Boime (D) *a and Johann Bouclé (DD *b
}

\begin{abstract}
This work presents an original nanostructured architecture for energy conversion applications based on vertically aligned carbon nanotubes (VACNTs). The developed approach consists in exploiting the directional charge transport provided by the VACNTs-based network to efficiently extract photogenerated electrons in perovskite solar cells. High density, $20 \mu \mathrm{m}$-long carpets of VACNTs were synthesised by an aerosol-assisted chemical vapor deposition (AACVD) process on aluminium substrates. The technique of electrodeposition, usually used for thin film elaboration, was adapted to decorate carbon nanotubes (CNTs) with ZnO nanoparticles (NPs) and reinforce the CNT scaffold. This procedure was successful even for the high CNT carpet density used here. Then, this dense nanostructured network was successfully infiltrated by a methylammonium lead iodide perovskite that crystallized homogeneously between the nanotubes and all along their length, from the bottom to the top. While this study is mainly focused on the material nano-engineering aspect, the charge extraction ability of the system was tested by photoluminescence spectroscopy. A quantitative luminescence quenching is demonstrated, evidencing an efficient charge transfer between the perovskite and the VACNTs/ZnO electrode. These promising results led to the fabrication of fully working devices that demonstrated a diode-like electrical response, characteristic of a solar cell in the dark. Considering both the possibility to develop this architecture at the industrial scale and the obtained physical properties, electrodes based on VACNTs decorated by ZnO NPs seem to be a relevant and promising candidate for efficient optoelectronic devices such as perovskite solar cells.
\end{abstract}

Received 14th April 2020,

Accepted 29th June 2020

DOI: $10.1039 / \mathrm{d} 0 \mathrm{ma00204f}$

rsc.li/materials-advances roll-to-roll processes. ${ }^{2}$ Nevertheless, research is still needed to improve the global PSC performance and stability. ${ }^{3}$ In particular, engineering charge extraction layers is crucial to ensure selective charge extraction and efficient current collection. Usually, electron collection is ensured by the use of a selective contact layer composed of semiconducting oxide such as $\mathrm{TiO}_{2}, \mathrm{ZnO}$ or $\mathrm{SnO}_{2}$ and one of the strategies explored in the literature to enhance the charge collection efficiency of PSCs is the addition of carbon nano-objects, such as graphene materials, in a small amount. ${ }^{4-7}$ Among these carbon nanoobjects, carbon nanotubes (CNTs) have sparked very active research since the 2000s in the case of polymer nanocomposites to improve mechanical and electrical properties, ${ }^{8}$ and particularly in the case of organic solar cells. ${ }^{9}$ For this latter application, the predominant idea was to exploit the photoinduced charge transfer between a conjugated polymer absorber and the CNTs whose anisotropy should favour the transport and the collection of the charges towards the electrodes. It follows from these studies that the exploitation of the anisotropy of the 
CNTs is mainly effective for charge transport only if these nanoparticles are suitably oriented perpendicular to the electrodes. ${ }^{10}$

Here, we propose an alternative strategy, which uses a network based on vertically aligned carbon nanotubes (VACNTs) as an electron extracting electrode. Such a simple system is a step in the quest for a fully directional electron transport, reducing the inherent charge recombination mechanisms associated with conventional isotropic electron transport layers. In the field of perovskite-based photovoltaics, the benefit of CNTs for charge transport has been reported in the literature. ${ }^{11-14}$ CNTs have been especially integrated in perovskite solar cells to improve hole collection, for instance as an interface between the perovskite and spiro-OMeTAD or directly mixed into the spiro-OMeTAD layer. ${ }^{12}$ A fast hole transfer from the perovskite to the nanotubes has been evidenced, leading to a reduction of charge recombination phenomena within the solar cells. ${ }^{15,16}$ An improvement of photovoltaic performance was observed, as well as a better stability of the PSCs. However, to date the use of vertically aligned carbon nanotubes (VACNTs) in perovskite solar cells has not been reported in the literature, yet VACNT growth presents the major advantage of being compatible with industrial development on large areas through roll-to-roll processes. ${ }^{17}$ The use of VACNTs has been reported in dyesensitized solar cells (DSSCs) most of the time to replace the expensive Pt electrode, ${ }^{18,19}$ although their use for electron collection is also reported..$^{20,21}$ We highlight here that in all cases reported in the literature based on vertically aligned VACNTs, the cells are composed of a liquid electrolyte, ${ }^{18-21}$ and therefore the dense carbon nanotube network infiltration by perovskite constitutes a real challenge, which is therefore a delicate and original part of our study. Thus, we present here the development of a VACNT based electrode infiltrated by the classic MAPI $\left(\mathrm{CH}_{3} \mathrm{NH}_{3} \mathrm{PbI}_{3}\right)$ reference perovskite, together with the demonstration of a working diode-like device. These promising results clearly broaden the horizons of carbon-based components in the field of halide perovskite optoelectronics.

\section{Experimental section}

This section describes the material synthesis, whereas details of the characterization methods are provided in the ESI. $\dagger$
Synthesis of vertically aligned carbon nanotubes (VACNTs) on an aluminium substrate

The VACNTs are synthesised with a one-step AACVD (aerosol assisted chemical vapor deposition) process developed on an aluminum substrate, using acetylene as a carbon precursor and ferrocene as a catalyst. ${ }^{17}$ This synthesis is conducted at $580{ }^{\circ} \mathrm{C}$ during 10 minutes. The $\mathrm{Fe} / \mathrm{C}$ ratio in the precursors is kept lower than $2 \mathrm{wt} \%$, and the total flow rate is maintained in the [100-333] sccm $\mathrm{min}^{-1}$ range while the acetylene and hydrogen concentration in the gas mixture is in the range of $10-20 \%$. Under these conditions, vertically-aligned multi-walled carbon nanotubes (MWCNTs) are obtained with a length of $20 \mu \mathrm{m}$ and a diameter of $10 \mathrm{~nm}$ (from TEM analyses) providing a specific surface area of $410 \mathrm{~m}^{2} \mathrm{~g}^{-1}$. The specificity of these VACNTs is their very high density, typically $\sim 10^{11}$ VACNTs per $\mathrm{cm}^{2}$, which brings the problem of conformal deposition of the $\mathrm{ZnO} /$ perovskite with a low intertube space of approximately $10 \mathrm{~nm}$.

\section{Electrodeposition parameters of ZnO nanoparticles on VACNTs}

The electrodeposition of ZnO nanoparticles along the VACNTs is performed using a three-electrode set-up. The sample Al/VACNTs correspond to the working electrode, whereas activated carbon is used as a counter-electrode and an SCE (saturated calomel electrode) is used as a reference. The electrolyte is an aqueous solution with $0.1 \mathrm{M}$ of $\mathrm{Li}\left(\mathrm{NO}_{3}\right)$ and $0.5 \mathrm{M}$ of $\mathrm{Zn}\left(\mathrm{NO}_{3}\right)_{2}$. The deposition is carried out by chronoamperometry with the application of $-1.1 \mathrm{~V}$ per SCE during 10 minutes. A short parametric study on the electrodeposition conditions led to the choice of these parameters to obtain an unprecedented and homogeneous association of electrodeposited $\mathrm{ZnO}$ with dense VACNT carpets. Samples are then annealed at $450{ }^{\circ} \mathrm{C}$ during 5 minutes to get rid of the residual component after electrodeposition.

\section{Perovskite deposition on the VACNTs(/ZnO)-based network}

The $\mathrm{CH}_{3} \mathrm{NH}_{3} \mathrm{PbI}_{3}$ perovskite (or MAPI) is deposited under ambient conditions (open air and at room temperature) with a two-step process illustrated in Fig. 1. First, $462 \mathrm{mg}$ of $\mathrm{PbI}_{2}$ is dissolved into $1 \mathrm{~mL}$ of DMF. The solution is heated at $70{ }^{\circ} \mathrm{C}$ and then, $10 \mu \mathrm{L}$ is drop-cast onto the substrate. An annealing at $70{ }^{\circ} \mathrm{C}$ for 30 minutes is carried out. To form the perovskite, the sample is then immersed into a solution of methylammonium iodide (100 mg of MAI dissolved into $10 \mathrm{~mL}$ of 2-propanol) for

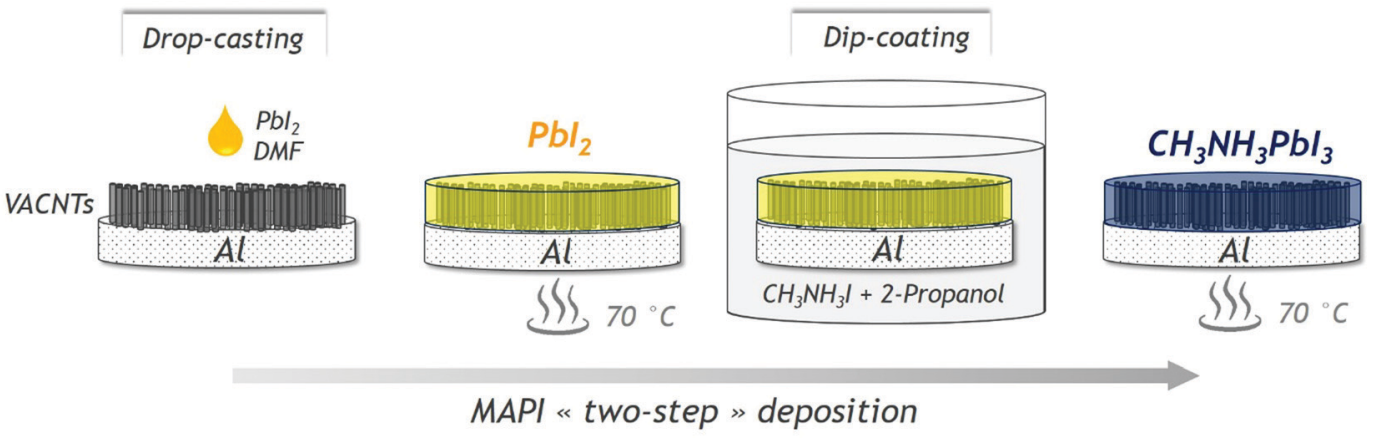

Fig. 1 Scheme of the two-step process used for MAPI deposition. 
30 seconds. Finally, the sample is annealed again at $70{ }^{\circ} \mathrm{C}$ for 30 minutes to ensure the MAPI crystallization.

\section{Results \& discussion}

\section{Electrodeposition of $\mathrm{ZnO}$ nanoparticles}

The work is focused here on carpets of $20 \mu \mathrm{m}$-long multi-walled carbon nanotubes vertically aligned with a high density of $10^{11}$ CNTs per $\mathrm{cm}^{2}$ on an aluminium substrate (12 $\mathrm{mm}$ diameter). Their synthesis, presented in the experimental part, is performed by aerosol-assisted chemical vapor deposition (AACVD), developed at low temperature. ${ }^{17}$ Our study revealed that the density of VACNTs is a key parameter to achieve high quality layers after perovskite deposition. Indeed, we emphasized that VACNT networks with a lower CNT density $\left(10^{9}\right.$ CNTs per $\left.\mathrm{cm}^{2}\right)$ are altered during the solvent evaporation occurring during the perovskite crystallization. A clear disruption of nanotube alignment is observed in this latter case (see example in the ESI, $\uparrow$ Fig. S1). Therefore, in addition to using denser VACNT networks, we propose to deposit nanocrystalline ZnO on the CNTs before the perovskite infiltration (as illustrated in Fig. 2a).
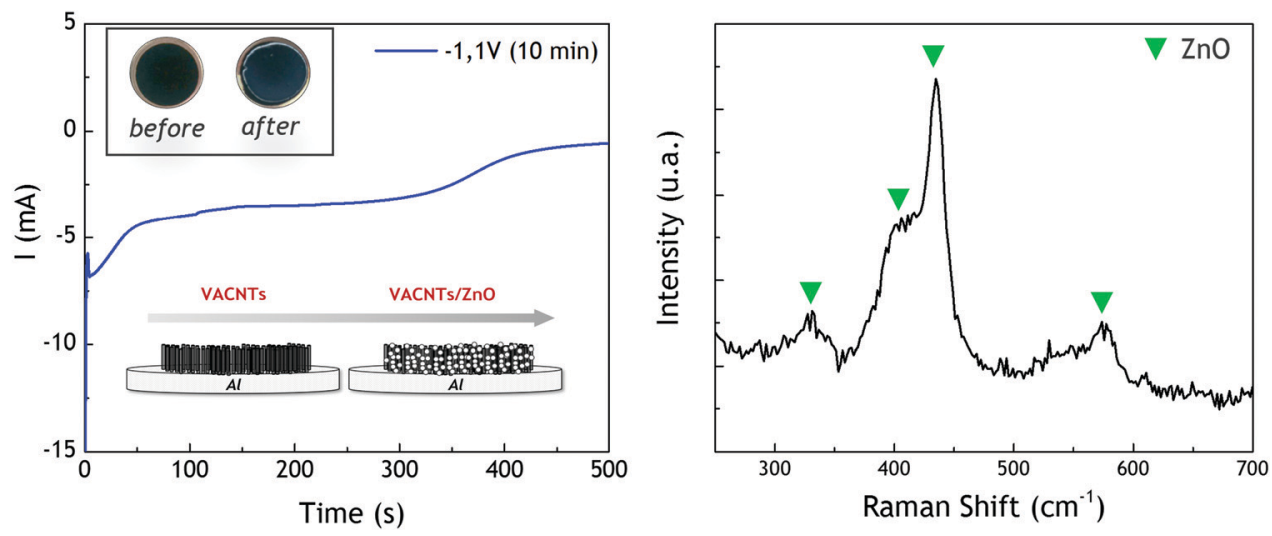

(a)

(b)
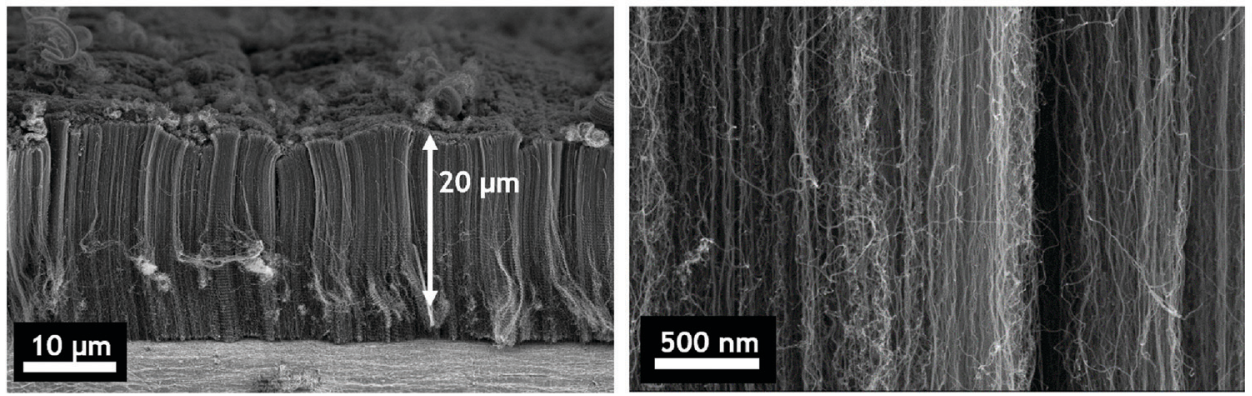

(c)

(d)
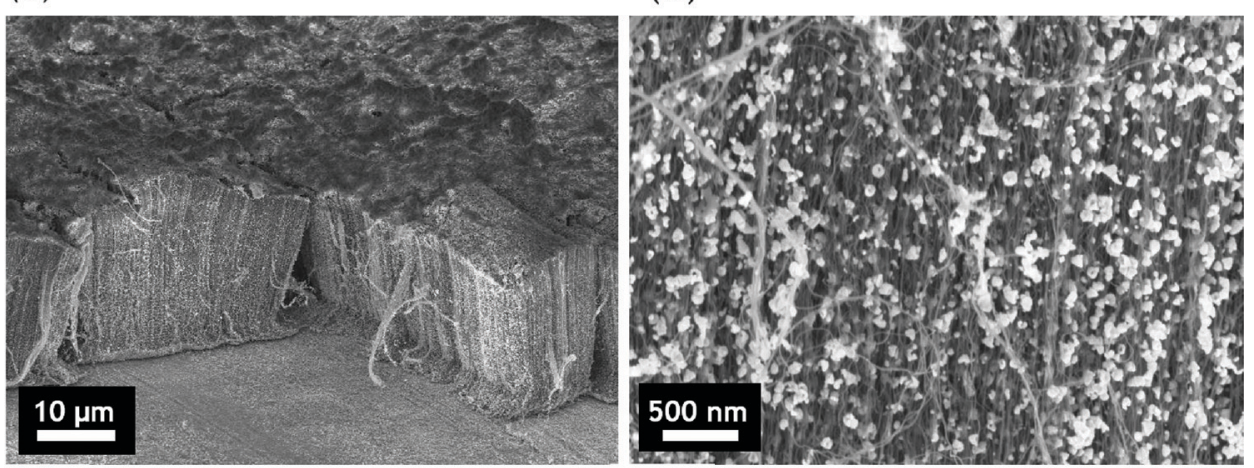

(e)

(f)

Fig. 2 (a) ZnO electrodeposition curve (chronoamperometry at $-1.1 \mathrm{~V}$ per SCE during 10 minutes) with the sample of Al/VACNTs ( $\varnothing 12 \mathrm{~mm}$ ) before and after $\mathrm{ZnO}$ electrodeposition. The insets show the aspect of the sample before and after electrodeposition. (b) Raman spectroscopy (532 nm LASER) of Al/VACNTs after ZnO electrodeposition (without thermal annealing). (c and d) SEM cross-section of high density Al/VACNTs. (e and f) SEM cross-section of Al/VACNT/ZnO nanocomposites at different magnifications. The presence of $\mathrm{ZnO}$ is clearly observed on the CNTs, whatever the position inside the carpet. 
Zinc oxide can indeed be used as an electron transport material in perovskite solar cells and presents also the advantage of blocking holes for a better charge separation. ${ }^{22} \mathrm{ZnO}$ was also recently demonstrated to be a very suitable interfacial material in association with various perovskite active layers, enabling high efficiencies. ${ }^{23}$ The literature reports $\mathrm{ZnO}$ deposition on VACNTs either by CVD (Chemical Vapor Deposition), by thermal evaporation, or by a hydrothermal process. ${ }^{20,21,24}$ Since the first electrodeposited $\mathrm{ZnO}$ film obtained by Izaki et al. in 1996, ZnO is easily elaborated as thin films by electrochemical deposition. ${ }^{25}$ Based on these results, we developed the electrodeposition of $\mathrm{ZnO}$ nanoparticles all along the dense aligned VACNT carpets. Properly tuning the experimental parameters of electrodeposition (see Experimental section), we present in Fig. 2(a) the typical chronoamperometric curve obtained during ZnO electrodeposition.

Inset in Fig. 2(a) shows the top view optical image of the sample before and after the experiment. Its darkness is due to the carbon nanotube carpet whereas a thin transparent material is observed after electrodeposition. The presence of $\mathrm{ZnO}$ is confirmed by Raman spectroscopy performed on the VACNT carpet after deposition, as shown in Fig. 2(b). The characteristic peaks of $\mathrm{ZnO}$ are detected at $405 \mathrm{~cm}^{-1}, 434 \mathrm{~cm}^{-1}$ and $575 \mathrm{~cm}^{-1}$ and correspond respectively to the $\mathrm{A}_{1 \mathrm{LO}}, \mathrm{E}_{2}$ and $\mathrm{E}_{1 \mathrm{LO}}$ modes. The principal peak at $434 \mathrm{~cm}^{-1}$ is related to the hexagonal wurtzite phase of $\mathrm{ZnO}$, which is also confirmed by X-ray diffraction (see the ESI, $\dagger$ Fig. S3), highlighting the preferred (101) and (100) orientations. These observations are consistent with the literature data for similar electrodeposition conditions. ${ }^{26,27}$

A thermal annealing ( 5 minutes at $450{ }^{\circ} \mathrm{C}$ ) is then systematically performed on the sample to ensure an optimal crystallization of the $\mathrm{ZnO}$ as well as removal of residual hydroxyl groups due to an electrodeposition process. The presence of such groups is indeed known to cause perovskite degradation. ${ }^{28,29} \mathrm{XRD}$ and Raman spectroscopy measurements after thermal annealing are provided in the ESI $\dagger$ (respectively Fig. S3 and S4). They both evidence, as expected, a significant improvement of the crystallinity of the deposited metal oxide nanoparticles under annealing.

Fig. 2(c) and (d) show the SEM cross-sections of the VACNT networks on the aluminium substrate, highlighting the good alignment degree of the nanotubes and the high CNT density of the carpet. Fig. 2(e) and (f) correspond to the VACNTs after the electrodeposition of $\mathrm{ZnO}$ (and after thermal annealing). In contrast with unprotected VACNTs (see Fig. S1, ESI $\dagger$ ), we clearly observe on Fig. 2(e) that the carbon nanotube alignment is still conserved after the $\mathrm{ZnO}$-based protection elaboration. In addition, we see in Fig. 2(f) that the $\mathrm{ZnO}$ is formed as spherical nanoparticles along the VACNTs. A top view of the sample is provided in Fig. S5 (see the ESI $\dagger$ ) and shows a relatively compact and very homogeneous covering of VACNTs by ZnO. Although some cracks remain visible on top of the sample, our methodology proves to be suitable to keep the alignment over a large area.

\section{Infiltration of perovskite within the VACNT/ZnO network}

The second part of our work consisted in studying the capability of the perovskite to infiltrate and crystallize in the VACNT network as well as the capability of the CNTs to keep their alignment during the perovskite infiltration, as illustrated in Fig. 3.

The reference methylammonium lead iodide perovskite is first deposited thanks to a two-step deposition method, described in the experimental part, on the Al/VACNT system. We emphasize that although a perovskite layer based on the methylammonium cation is known to generate a less stable interface with $\mathrm{ZnO},{ }^{30}$ this reference compound remains largely investigated in the field, and appears to be a relevant first choice considering our two-step deposition method. Several approaches were indeed investigated during a preliminary study using different perovskite solutions, or different steps for perovskite deposition, in order to optimize the process and obtain the best perovskite infiltration into the entire dense VACNTs carpet on Al. Details of this study are presented in the ESI $\dagger$ (see Fig. S2). After the two-step MAPI deposition, we observe a good infiltration and crystallization of perovskite all along the VACNTs, from the bottom to the top (see the ESI, $\dagger$ Fig. S6(c)). However, a slight densification of the VACNTs is observed, as a consequence of the solvent evaporation phase which tends to aggregate the nanotubes, in agreement with the literature. ${ }^{31-34}$ As a result, undesirable cavities are present in the Al/VACNTs/perovskite sample (see the ESI, $\dagger$ Fig. S6(a)), leading to a strong inhomogeneity in the sample. To prevent this issue, the perovskite infiltration process has been preferably applied to the VACNT/ZnO network. Fig. 4(a)-(c) are crosssection views, at different magnifications, of the Al/VACNTs/ $\mathrm{ZnO} /$ perovskite nanocomposite, whereas image (d) is a top view. In the presence of the electrodeposited $\mathrm{ZnO}$, we clearly identify crystallized MAPI grains mixed with the ZnO particles all along the VACNTs. Their cubic shape is typical from the grains obtained with a two-step deposition process. ${ }^{35}$ Therefore, we assume that our scaffold structure does not significantly influence the perovskite crystallization. Moreover, it has been shown in the literature that this process is appropriate especially when perovskite is deposited onto porous materials, ${ }^{36}$ which reinforces

\section{VACNTs}

VACNTs/ZnO

VACNTs/ZnO/Perovskite

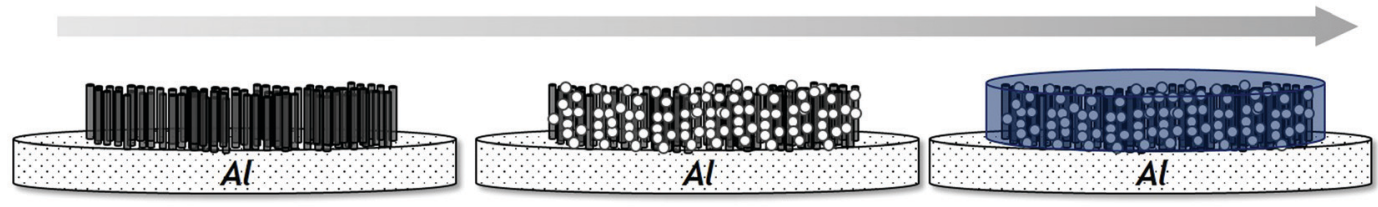

Fig. 3 Scheme of the elaboration process developed for perovskite infiltration into the VACNT/ZnO network. 


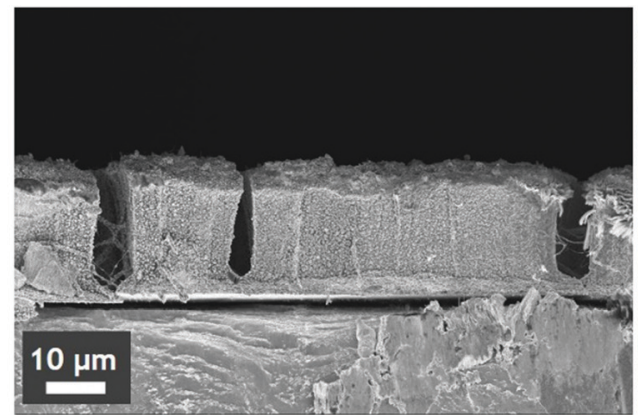

(a)

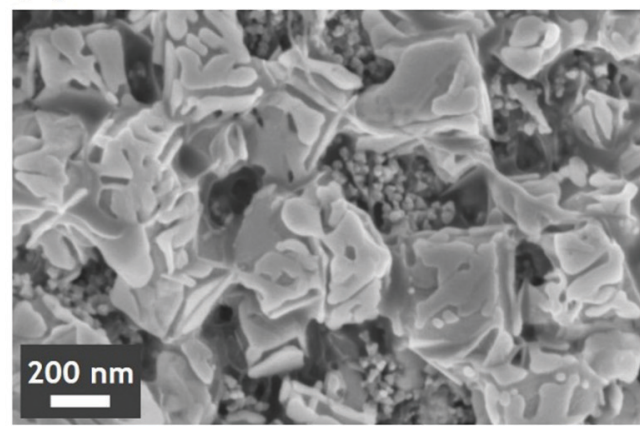

(c)

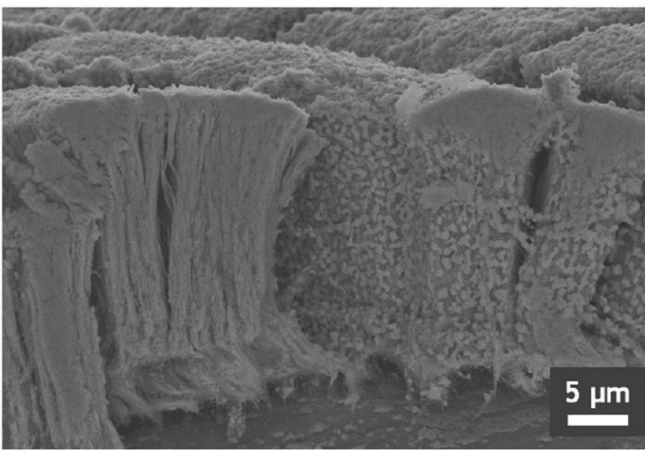

(e)

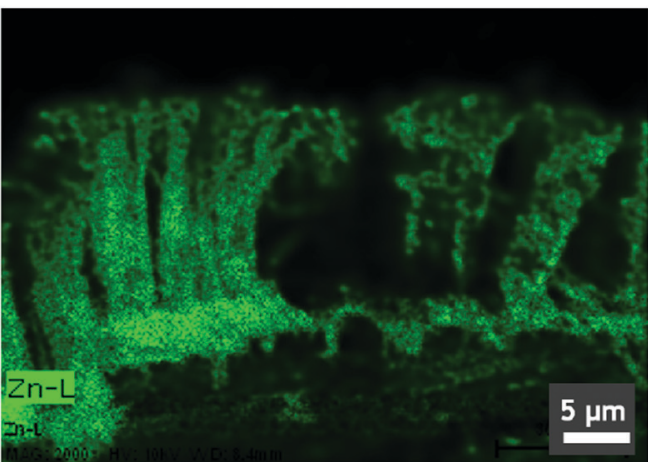

(g)

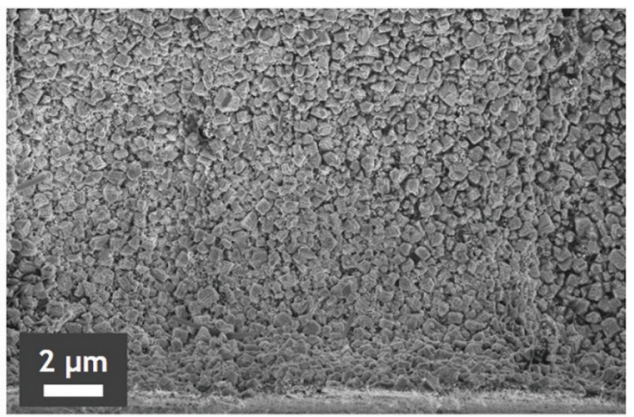

(b)

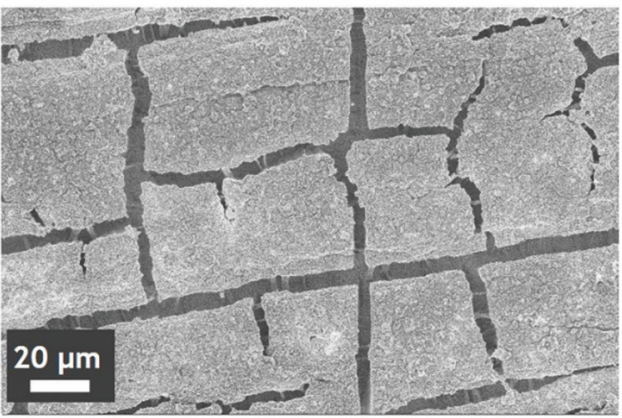

(d)

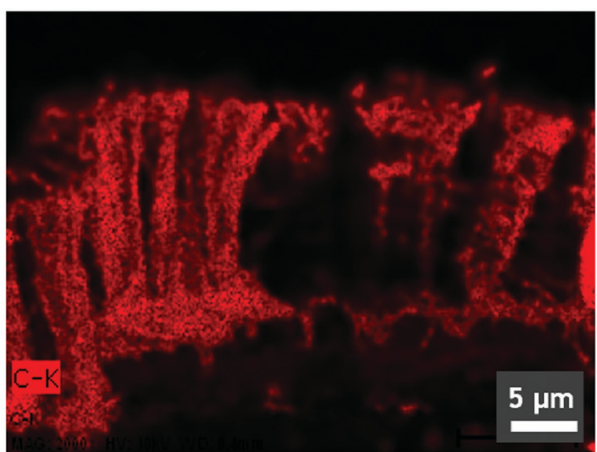

(f)

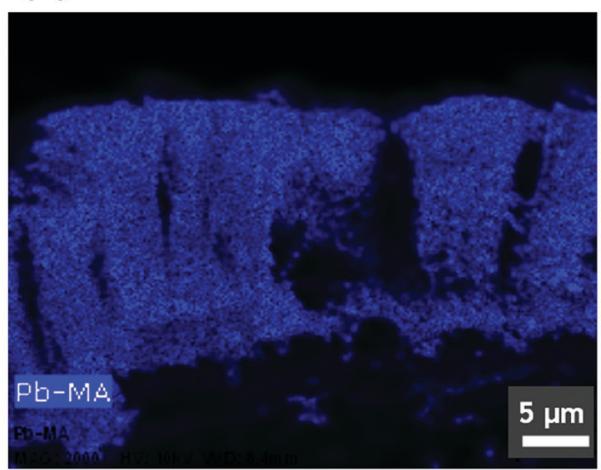

(h)

Fig. 4 (a-c) SEM cross-section views at different magnifications of AI/VACNT/ZnO/perovskites and (d) top view of the deposit. (e) SEM cross-section of Al/VACNTs/ZnO/perovskite and associated EDX analysis. (f) Carbon repartition, (g) Zn repartition and (h) Pb repartition. These pictures show the efficient colocalization of CNTs, ZnO and perovskite.

the consistence of our results. The other main point here is the preservation of CNT alignment. Despite the low available intertube space (due to high density of CNTs combined with the ZnO nanoparticle presence), we managed to crystallize the perovskite all along the carpet, from the bottom to the top. This system is also much more compact than without $\mathrm{ZnO}$ (see Fig. S6 in the 
$\mathrm{ESI} \dagger$ for comparison with and without $\mathrm{ZnO}$ ). The quality of the infiltration is moreover confirmed by energy dispersive X-ray (EDX) spectroscopy mapping analysis presented in Fig. 4(e)-(h). The carbon repartition is highlighted in red in Fig. 4(f). The repartition of zinc atoms (green channel in Fig. 4(g)) perfectly matches with the $\mathrm{C}$ repartition, demonstrating the homogeneous $\mathrm{ZnO}$ electrodeposition from the bottom to the top of the CNTs. Finally, the repartition of $\mathrm{Pb}$, which is linked to the presence of perovskite, is presented in blue in Fig. 4(h). The presence of $\mathrm{Pb}$ in every area of the sample proves here the successful infiltration of perovskite and the homogeneity of the nanocomposite sample. We finally emphasize that our protocol for perovskite infiltration is not optimized for these early stages, and that it will be associated with a large fraction of defect states. Consequently, passivation strategies or protocols for achieving larger perovskite grain size will be naturally considered towards the improvement of the active layer, as reported for the most efficient perovskite solar cells. $^{37}$

\section{From the electrode to the device}

In order to evaluate to potential of the $\mathrm{Al} / \mathrm{VACNT} / \mathrm{ZnO} /$ perovskite systems for photovoltaic applications, we performed a first photophysical diagnostic using photoluminescence spectroscopy (PL). Thus, after the perovskite deposition, the sample was tested by photoluminescence spectroscopy under steady-state conditions and compared to $\mathrm{Al} /$ perovskite and $\mathrm{Al} / \mathrm{VACNT} /$ perovskite reference samples elaborated simultaneously and under similar conditions. The measured absolute PL intensities are presented in Fig. 5 as a function of the emission wavelength. In the case of perovskite directly deposited on aluminium, the emission peak is centred at $782 \mathrm{~nm}$ corresponding to a direct optical transition at $1.59 \mathrm{eV}$, which is typical of the MAPI reference.

The perovskite emission is significantly quenched in the case of $\mathrm{Al} / \mathrm{VACNT} /$ perovskite samples, but is even more pronounced for the ZnO-containing sample, demonstrating an efficient charge transfer from the perovskite to the VACNT electrodes. This first result is then very promising for use of this nanostructured scaffold as an efficient electrode for electron collection in perovskite solar cells.

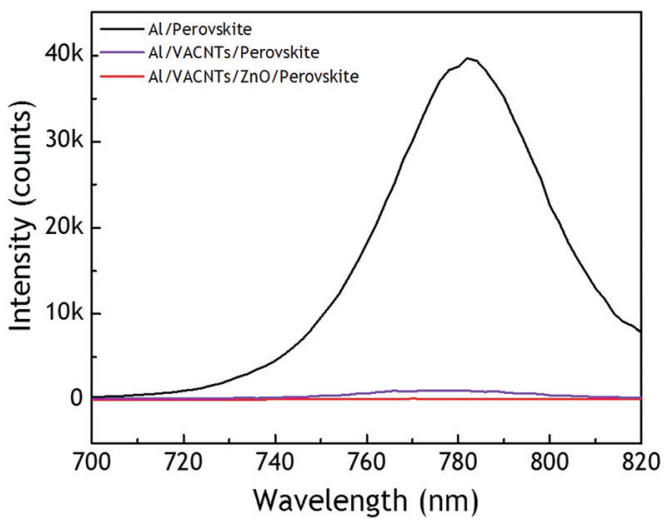

Fig. 5 Steady-state photoluminescence spectroscopy (exc. $470 \mathrm{~nm}$ ) of $\mathrm{Al} /$ perovskite, Al/VACNT/perovskites, and Al/VACNT/ZnO/perovskite samples.
Despite the relative difficulty to directly shift to a fully optimized perovskite solar cell due to the relative inhomogeneity of our system over a large area (Fig. 4(d)), we decided, as a proof of concept, to test the electrical behaviour of a simplified device to illustrate the potentialities of our system. Shifting to perovskite solar cells, a main issue relates to the nature of the growth substrate used for the synthesis of VACNTs. Indeed, third generation solar cells are mainly based on transparent conductive oxide (TCO) electrodes, such as indium tin oxide (ITO) or fluorinated tin oxide (FTO). The current status of our VACNT synthetic procedure by CVD is performed at $580{ }^{\circ} \mathrm{C}$, and is therefore not compatible with classical TCO deposited on glass. However, growing VACNTs at temperatures below $500{ }^{\circ} \mathrm{C}$ was reported using more complex methods, such as CVD assisted by hot filaments. ${ }^{38}$ The mechanical transfer of the VACNT layer from the growth substrate to secondary substrates presenting lower thermal stabilities was also reported in some cases. ${ }^{39,40}$ Although such methods are not the prime objective of this work, they will be highly relevant for shifting our proof of concept on TCO substrates. Therefore, we deposited a classic hole transport material, spiro-OMeTAD, on top of the perovskiteinfiltrated electrode. Details of the deposition procedure are provided in the ESI $\dagger$ (Fig. S7) as well as a comparison of the present study with a ZnO-free sample (see Section 3, Fig. S8 and S9, ESI $\dagger$ ), highlighting the relevance of covering VACNTs by ZnO nanoparticles. After the spiro-OMeTAD deposition, a homogeneous and compact sample is obtained (see Fig. S8(d) and (e) in the ESI $\dagger$ ). The final step of device processing corresponds to the deposition of a top electrode for hole collection. As mentioned previously, the relative inhomogeneity of our initial architecture over a large area prevents, at this early stage, the use of conventional vacuum deposited metallic electrodes (such as gold), which would be associated with large current leakages. We thus simply use here a drop of gallium/indium (EGa:In) eutectic alloy, which presents the advantage of being liquid at ambient temperature, deposited on top of the $\mathrm{Al} / \mathrm{VACNTs} / \mathrm{ZnO} /$ perovskite/ spiro-OMeTAD system. The use of this liquid top electrode enables the formation of a quite small active area, limiting the risk for electrical shunts by direct contacts between the top and VACNTs/Al electrode. Basic current-voltage measurements were then performed using standard contact tips connected to a source-measure unit. A picture of the set-up with the sample is presented in the inset of Fig. 6.

The device was tested under dark conditions and the corresponding $I-V$ curve is shown in Fig. 6 , in both linear and semilogarithmic scale. As we can see, a diode-like current evolution is observed, with a turn-on voltage on the order of $0.7 \mathrm{~V}$. In the logarithmic representation, a rectification factor of $\sim 4$ (at $\pm 1 \mathrm{~V}$ ) is evidenced for the device based on the $\mathrm{ZnO}$ coating, whereas an ohmic behaviour is observed for a $\mathrm{ZnO}$-free device (see the ESI, $\dagger$ Fig. S9). These first measurements confirm the relevance of our strategy towards the demonstration of promising charge extraction electrodes based on vertically aligned carbon nanotube networks of potential interest for perovskite solar cells.

From this point, two questions remain to be addressed regarding device operation: the nature of charge transport 


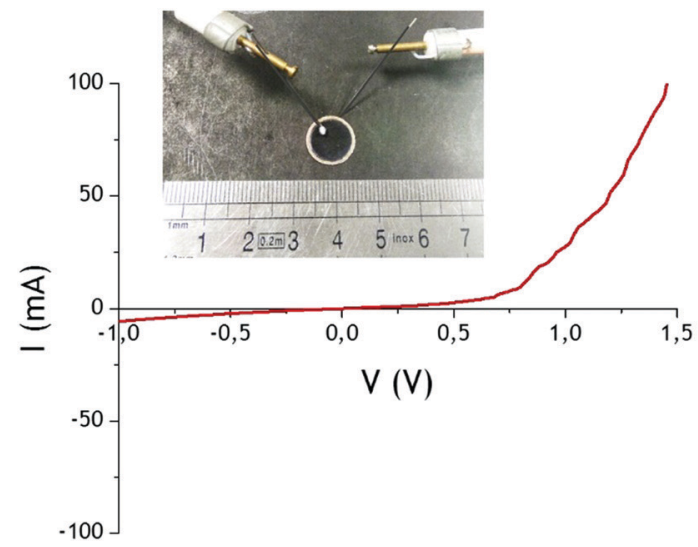

(a)

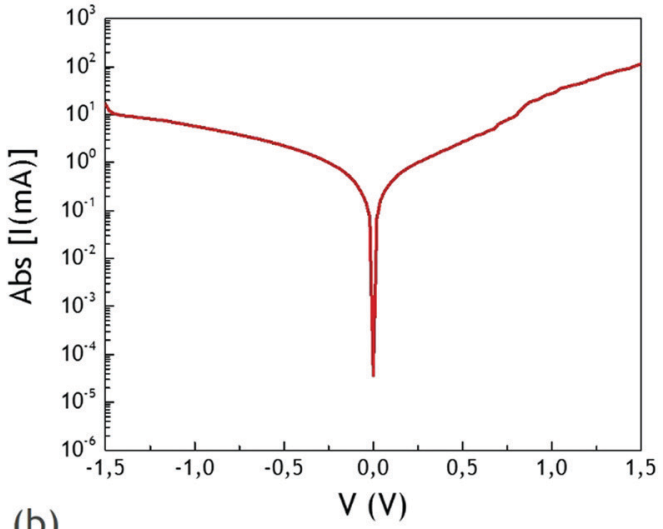

(b)

Fig. 6 (a) I-V dark measurment of Al/VACNTs/ZnO/perovskite/spiro-OMeTAD/EGa:In device $-0.0079 \mathrm{~cm}^{2}$ active area and (b) semi-logarithmic representation.

properties associated with the VACNTs and the impact of the ZnO coating on these properties. First, since carbon nanotubes are multiwall (MWCNTs), they are expected to exhibit a metallic character rather than a semiconducting behaviour. ${ }^{41}$ To support this assumption, levels of conductivity of vertical MWCNTs from VACNT carpets have been estimated in the order of 2 to $6 \times 10^{5} \mathrm{~S} \mathrm{~m}^{-1}{ }^{42,43}$ Therefore, without $\mathrm{ZnO}$ at their surface, the aligned nanotube network will not play a main role in charge selectivity, and will more likely play the role of a collecting electrode rather than a classical charge extraction layer. This feature is also responsible for the large leakage currents observed in devices where direct contacts between the VACNTs and the HTM are important (as observed through the purely ohmic behaviour of devices processed without zinc oxide, see Fig. S9 in the ESI $\dagger$ ). As commonly stated, particularly in the case of organic solar cells where carbon nanotubes have been incorporated into the active layer, the charges drained by the nanotubes depend greatly on the transport properties of the surrounding material. For example, their conductivity is more likely p-type in the case of P3HT-NTC-PCBM ternary composites, for which the most intimate mixture with the nanotubes is provided by P3HT. ${ }^{44}$ It was also similarly observed in the case of perovskite solar cells using CNTs in association with p-type organic species such as spiro-OMeTAD for improved hole collection. ${ }^{12}$ Conversely, CNTs grafted with fullerenes show n-type transport in the same P3HT-NTC-PCBM blend. ${ }^{45}$ In our case, when $\mathrm{ZnO}$ is present at the VACNT surface, a clear rectifying behaviour of the obtained diodes is obtained. The presence of the metal oxide at the bottom of the carpet also prevents direct shunts between the aluminium substrate and the spiro-OMeTAD HTM. Considering a conventional n-type character for $\mathrm{ZnO}$, which is largely used as an electron transport layer in third generation solar cells, we believe that our ZnOcoated VACNT forests will selectively drain electrons to the aluminium conductive substrate. The use of large specific area electrodes and the successful coating by $\mathrm{ZnO}$ ensure an efficient charge extraction is this case. We still emphasize that a significant contribution from a non-ohmic Al/VACNT interface remains possible at this stage. Moreover, the presence of $\mathrm{ZnO}$ at the CNT surface is also expected to modify their intrinsic charge transport properties, as previously observed for MWCNTs grafted by ZnO nanoparticles. ${ }^{46}$ Further investigations will be required to suitably address these issues. In addition, additional tests under illumination could be worth considering after carefully optimizing the global homogeneity of the VACNT electrode, through the deposition of a transparent top-electrode based on silver nanowires or graphene for instance,${ }^{47-49}$ that was not yet tested in the framework of this first study (the nature of our bottom and top electrodes are not compatible with electrical tests under standard AM1.5G illumination). Finally, the exact influence of the ZnO particles at the VACNT/perovskite interface is also of particular interest for future studies.

\section{Conclusions}

This work started with the motivation of developing a perovskite photovoltaic device based on vertically aligned carbon nanotubes (VACNTs) to favour a directional transport of photo-generated electrons. To do so, we elaborated electrodes and studied the influence of the VACNT density, the importance of the perovskite deposition method as well as the influence of the presence of a structuring intermediate material. Through this study, ZnO nanoparticles were successfully deposited by chemical electrodeposition along CNTs inside a dense VACNT carpet. In addition, we show that a reference halide perovskite such as MAPI can efficiently infiltrate a dense VACNT carpet and crystallize without altering the CNT alignment thanks to the presence of ZnO nanoparticles. Preliminary diode-like devices based on a molecular hole transport material indicate the relevance of this architecture. The key point here is the combination of the high CNT density with the presence of $\mathrm{ZnO}$ nanoparticles. Another important advantage of this architecture lies in the fact that these three steps - VACNT synthesis, ZnO nanoparticle 
electrodeposition and perovskite deposition - are compatible with industrial large-scale processes. Our results demonstrated that this nanostructured scaffold presents promising physical properties which could allow efficient charge carrier collection or injection in a large variety of devices such as solar cells, photoelectrochemical cells, or photocatalytic systems.

\section{Conflicts of interest}

There are no conflicts to declare.

\section{Acknowledgements}

We are grateful for the financial support from IDEX Paris-Saclay and from the SigmaLim LabEX in Limoges. JB acknowledge the French Research National Agency (ANR) through the project "SuperSansPlomb" (ANR-15-CE05-0023-03), as well as the PLATINOM technology platform of the University of Limoges. RB, NHB and JB acknowledge Richard Mayet and the CARMALIM common service of the University of Limoges for XRD analysis.

\section{References}

1 NREL, Best Research-Cell Efficiency Chart, https:/www.nrel. gov/pv/cell-efficiency.html, accessed June 3, 2020.

2 Z. Li, T. R. Klein, D. H. Kim, M. Yang, J. J. Berry, M. F. A. M. van Hest and K. Zhu, Nat. Rev. Mater., 2018, 3, 18017.

3 L. Qiu, L. K. Ono and Y. Qi, Mater. Today Energy, 2018, 7, 169-189.

4 Z. Zhang, L. Wei, X. Qin and Y. Li, Nano Energy, 2015, 15, 490-522.

5 E. L. Lim, C. C. Yap, M. H. H. Jumali, M. A. M. Teridi and C. H. Teh, Nano-Micro Lett., 2018, 10, 27.

6 J. Bouclé and N. Herlin-Boime, Synth. Met., 2016, 222, 3-16.

7 R. Belchi, A. Habert, E. Foy, A. Gheno, S. Vedraine, R. Antony, B. Ratier, J. Bouclé and N. Herlin-boime, ACS Omega, 2019, 4, 11906-11913.

8 M. Jouni, D. Djurado, V. Massardier and G. Boiteux, Polym. Int., 2017, 66, 1237-1251.

9 B. Ratier, J.-M. Nunzi, M. Aldissi, T. M. Kraft and E. Buncle, Polym. Int., 2012, 61, 342-354.

10 R. Radbeh, E. Parbaile, M. Chakaroun, B. Ratier, M. Aldissi and A. Moliton, Polym. Int., 2010, 59, 1514-1519.

11 M. Bag, L. A. Renna, S. P. Jeong, X. Han, C. L. Cutting, D. Maroudas and D. Venkataraman, Chem. Phys. Lett., 2016, 662, 35-41.

12 S. N. Habisreutinger, R. J. Nicholas and H. J. Snaith, Adv. Sci. News, 2017, 7, 1601839.

13 N. Ahn, I. Jeon, J. Yoon, E. I. Kauppinen, Y. Matsuo, S. Maruyama and M. Choi, J. Mater. Chem. A, 2018, 6, 1382-1389.

14 I. Jeon, S. Seo, Y. Sato, C. Delacou, A. Anisimov, K. Suenaga, E. I. Kauppinen, S. Maruyama and Y. Matsuo, J. Phys. Chem. C, 2017, 121, 25743-25749.

15 P. Schulz, A.-M. Dowgiallo, M. Yang, K. Zhu, J. L. Blackburn and J. J. Berry, J. Phys. Chem. Lett., 2016, 7, 418-425.
16 R. Ihly, A. Dowgiallo, M. Yang, P. Schulz, N. J. Stanton, O. G. Reid, A. J. Ferguson, K. Zhu, J. J. Berry and J. L. Blackburn, Energy Environ. Sci., 2016, 9, 1439-1449.

17 F. Nassoy, M. Pinault, J. Descarpentries, T. Vignal, P. Banet, P.-E. Coulon, T. Goislard de Monsabert, H. Hauf, P.-H. Aubert, C. Reynaud and M. Mayne-L'Hermite, Nanomaterials, 2019, 9, 1590.

18 H. Anwar, A. E. George and I. G. Hill, Sol. Energy, 2013, 88, 129-136.

19 P. Dong, C. L. Pint, M. Hainey, F. Mirri, Y. Zhan, J. Zhang, M. Pasquali, R. H. Hauge, R. Verduzco, M. Jiang, H. Lin and J. Lou, ACS Appl. Mater. Interfaces, 2011, 3, 3157-3161.

20 J. Chen, C. Li, D. W. Zhao, W. Lei, Y. Zhang, M. T. Cole, D. P. Chu, B. P. Wang, Y. P. Cui, X. W. Sun and W. I. Milne, Electrochem. Commun., 2010, 12, 1432-1435.

21 G. Y. Zeng, K. S. Nian and K. Y. Lee, Diamond Relat. Mater., 2010, 19, 1457-1460.

22 L.-C. Chen and Z.-L. Tseng, Nanostructured Solar Cells, ed. N. Das, IntechOpen, 2017, DOI: 10.5772/65056.

23 D. Zheng, G. Wang, W. Huang, B. Wang, W. Ke, J. L. Logsdon, H. Wang, Z. Wang, W. Zhu, J. Yu, M. R. Wasielewski, M. G. Kanatzidis, T. J. Marks and A. Facchetti, Adv. Funct. Mater., 2019, 29, 1900265.

24 S. Parveen, A. Kumar, S. Husain, J. Ali, M. Zulfequar, M. Harsh and M. Husain, Mater. Res. Bull., 2016, 83, 12-18. 25 M. Izaki and O. Takashi, Appl. Phys. Lett., 1996, 68, 2439.

26 M. Kumar and C. Sasikumar, Am. J. Mater. Sci. Eng., 2014, 2, 18-23.

27 L. I. Junwei, L. I. U. Zhifeng, E. Lei and Z. H. U. Zhichen, J. Wuhan Univ. Technol., Mater. Sci. Ed., 2011, 26, 47-51.

28 Y. Cheng, Q. Yang, J. Xiao, Q. Xue, H. Li, Z. Guan, H. Yip and S. Tsang, ACS Appl. Mater. Interfaces, 2015, 7, 19986-19993.

29 J. Yang, B. D. Siempelkamp, E. Mosconi, F. De Angelis and T. L. Kelly, Chem. Mater., 2015, 27, 4229-4236.

30 K. Schutt, P. K. Nayak, A. J. Ramadan, B. Wenger, Y.-H. Lin and H. J. Snaith, Adv. Funct. Mater., 2019, 29, 1900466.

31 Y. Yang, W. Yu, S. Wang and L. Zhang, Sol. Energy Mater. Sol. Cells, 2017, 161, 444-448.

32 K. Zhang, T. Li, L. Ling, H. Lu, L. Tang, C. Li, L. Wang and Y. Yao, Carbon, 2017, 114, 435-440.

33 Q. Li, R. Depaula, X. Zhang, L. Zheng, P. N. Arendt, F. M. Mueller, Y. T. Zhu and Y. Tu, Nanotechnology, 2006, 17, 4533-4536.

34 N. Chakrapani, B. Wei, A. Carrillo, P. M. Ajayan and R. S. Kane, Proc. Natl. Acad. Sci. U. S. A., 2004, 101, 4009-4012. 35 J. Lee and N. Park, MRS Bull., 2015, 40, 654-659.

36 J. Im, H. Kim and N. Park, APL Mater., 2014, 2, 081510.

37 Q. Jiang, Y. Zhao, X. Zhang, X. Yang, Y. Chen, Z. Chu, Q. Ye, X. Li, Z. Yin and J. You, Nat. Photonics, 2019, 13, 460-466.

38 S. Liatard, K. Benhamouda, A. Fournier, R. Ramos, C. Barchasz and J. Dijon, Chem. Commun., 2015, 51, 7749-7752.

39 J. L. Abot, Y. Song, M. J. Schulz and V. N. Shanov, Compos. Sci. Technol., 2008, 68, 2755-2760.

40 C. Lim, Y. Shin, S. Hong, S. Lee and D.-H. Kim, Adv. Mater. Interfaces, 2020, 7, 1902170. 
41 V. Skákalová, A. B. Kaiser, Y.-S. Woo and S. Roth, Phys. Rev. B: Condens. Matter Mater. Phys., 2006, 74, 085403.

42 C. Villeneuve, S. Pacchini, P. Boulanger, A. Brouzes, F. Roussel, M. Pinault, M. Mayne-L'Hermite and R. Plana, J. Appl. Phys., 2012, 112, 084327.

43 S. Amni, et al., CIGRÉ 2017 Colloquium proceeding, 2017.

44 S. Berson, R. de Bettignies, S. Bailly, S. Guillerez and B. Jousselme, Adv. Funct. Mater., 2007, 17, 3363-3370.

45 C. Li, Y. Chen, Y. Wang, Z. Iqbal, M. Chhowalla and S. Mitra, J. Mater. Chem., 2007, 17, 2406-2411.
46 I. Sameera, R. Bhatia and V. Prasad, Phys. B, 2010, 405, 1709-1714.

47 F. Guo, H. Azimi, Y. Hou, T. Przybilla, M. Hu, C. Bronnbauer, S. Langner, E. Spiecker, K. Forberich and C. J. Brabec, Nanoscale, 2015, 7, 1642-1649.

48 M. Chalh, S. Vedraine, B. Lucas and B. Ratier, Sol. Energy Mater. Sol. Cells, 2016, 152, 34-41.

49 N. N. Rosli, M. A. Ibrahim, N. Ahmad Ludin, M. A. Mat Teridi and K. Sopian, Renewable Sustainable Energy Rev., 2019, 99, 83-99. 